\title{
Does probabilism solve the great quantum mystery?
}

\author{
Nicholas MAXWELL
}

BIBLID [0495-4548 (2004) 19: 51; pp. 321-336]

\begin{abstract}
What sort of entities are electrons, photons and atoms given their wave-like and particle-like properties? Is nature fundamentally deterministic or probabilistic? Orthodox quantum theory (OQT) evades answering these two basic questions by being a theory about the results of performing measurements on quantum systems. But this evasion results in OQT being a seriously defective theory. A rival, somewhat ignored strategy is to conjecture that the quantum domain is fundamentally probabilistic. This means quantum entities, interacting with one another probabilistically, must differ radically from the entities of deterministic classical physics, the classical wave or particle. It becomes possible to conceive of quantum entities as a new kind of fundamentally probabilistic entity, the "propensiton", neither wave nor particle. A fully micro realistic, testable rival to OQT results.
\end{abstract}

Keywords: philosophy of science, quantum theory, realism, probabilism, wave/particle dilemma, propensities, measurement.

\section{Orthodox quantum theory is the best and worst of theories}

What sort of entities are electrons, photons and atoms given their wave-like and particle-like properties? Is nature fundamentally deterministic or probabilistic? Any decent theory of the quantum domain, able to provide us with genuine knowledge and understanding of its nature, ought to provide answers to these childishly elementary questions. Orthodox quantum theory (OQT) evades answering these questions by being a theory, not about quantum systems as such, but rather about the results of performing measurements on such systems. ${ }^{1}$

This state of affairs came about as follows. Bohr, Heisenberg, Dirac and the other creators of OQT did not know how to solve the quantum wave/particle dilemma. This created a grave problem for those seeking to develop quantum theory. How can one develop a consistent theory about entities that seem to be both wave-like and particle-like, as in the two slit experiment for example? Heisenberg around 1925 hit upon the strategy of evading this fundamental dilemma by developing what subsequently became matrix mechanics as a theory exclusively about the results of performing measurements on quantum systems, this version of quantum theory thus not needing to specify the nature of quantum systems when not undergoing measurement. Schrödinger, a little later in 1926, developed wave mechanics in the hope that it would be a precise theory about the nature of quantum systems. This theory, Schrödinger hoped, would show the electron to be wave-like in character. But then Born successfully interpreted the $\Psi$ function of Schrödinger's wave mechanics as specifying the probability of detecting the particle in question. According to Born's crucial interpre-

\footnotetext{
${ }^{1}$ Good introductory accounts of OQT, increasingly technical, are Squires (1986); Gillespie (1973); Feyn-
} man et al (1965). See also Maxwell (1998, appendix). 
tative postulate, $|\Psi|^{2} . \mathrm{dV}$ gives the probability of detecting the particle in volume element $\mathrm{dV}$ if a position measurement is performed. Schrödinger proved that his theory and Heisenberg's matrix mechanics are equivalent: the outcome, a sort of synthesis of the two theories, is OQT.

OQT is an extraordinarily successful theory empirically, perhaps the most successful in the whole of physics when one takes into account the range, immense diversity, and accuracy of its predictions. But not only does it fail to solve the great quantum mystery of what sort of entities electrons and atoms can be in view of their apparently contradictory particle and wave properties. It also fails to answer the other childishly elementary question: Is the quantum domain deterministic or probabilistic? The basic dynamic equation of OQT, Schrödinger's time-dependent equation, is deterministic in character. It tells us that quantum states, given by $\Psi$, evolve deterministically in time, as long as no measurements are made. But this does not mean OQT asserts that the quantum domain is deterministic. First, given OQT, $\Psi$ cannot be interpreted as specifying the actual physical state of a quantum system, just because OQT fails to solve the wave/particle dilemma, and thus fails to provide a consistent specification of the physical nature of quantum systems when not being measured. Given OQT, $\Psi$ must be interpreted as containing no more than information about the outcome of performing measurements. Secondly, OQT in general makes probabilistic predictions about the outcome of performing measurements, not (apart from exceptional circumstances) deterministic predictions. But one cannot conclude from this that OQT asserts that the quantum domain is fundamentally probabilistic in character, some physical states of affairs only determining what occurs subsequently only probabilistically. This is because, according to OQT, probabilistic outcomes only occur when we intervene, and make a measurement. In the absence of measurement, nothing probabilistic occurs at all, according to OQT. Indeed, if the process of measurement is treated quantum mechanically, then nothing probabilistic occurs at all, precisely because the basic dynamic equation of OQT, Schrödinger's time-dependent equation, is deterministic.

The inability of OQT to answer these two elementary questions is in itself a serious failure of the theory. But there are, as a consequence, a host of further failures and defects. Because OQT is about the results of performing measurements on quantum systems (and not about quantum systems per se, due to its failure to solve the wave/particle problem), in order to come up with physical predictions OQT must consist of two parts, (1) quantum postulates, and (2) some part of classical physics for a treatment of measurement. (2) is indispensable. (1) alone, precisely because OQT lacks its own quantum ontology, cannot predict anything physical at all —or at least can only make conditional predictions of the form: if such and such a measurement is made, such and such will be the outcome with such and such a probability. Thus $\mathrm{OQT}=\mathrm{QP}+\mathrm{CP}$, where "QP" stands for the quantum mechanical postulates of the theory, and "CP" stands for the classical postulates, required for measurement.

In what follows, a quantum "measurement" is a process that actually detects quantum systems; a process which prepares a quantum system to be in a certain quantum state, but does not detect the system, is a "preparation" rather than a "measurement". 
OQT, construed as QP + CP, as it must be, is a seriously defective theory. (a) OQT is imprecise, due to the inherent lack of precision of the notion of "measurement". How complex and macroscopic must a process be before it becomes a measurement? Does the dissociation of one molecule amount to a measurement? Or must a thousand or a million molecules be dissociated before a measurement has been made? Or must a human being observe the result? No precise answer is forthcoming. (b) OQT is ambiguous, in that if the measuring process is treated as a measurement, the outcome is in general probabilistic, but if this process is treated quantum mechanically, the outcome is deterministic. (c) OQT is very seriously ad hoc, in that it consists of two incompatible, conceptually clashing parts, QP and CP. OQT only avoids being a straightforward contradiction by specifying, in an arbitrary, ad hoc way, that QP applies to the quantum system up to the moment of measurement, and CP applies to the final measurement result. (d) OQT is non-explanatory, in part because it is ad hoc, and no ad hoc theory is fully explanatory, in part because OQT must presuppose some part of what it should explain, namely classical physics. OQT cannot fully explain how classical phenomena emerge from quantum phenomena because some part of classical physics must be presupposed for measurement. (e) OQT is limited in scope in that it cannot, strictly speaking, be applied to the early universe in conditions which lacked preparation and measurement devices. Strictly speaking, indeed, it can only be applied if physicists are around to make measurements. (f) OQT is limited in scope in that it cannot be applied to the cosmos as a whole, since this would require preparation and measurement devices that are outside the cosmos, which is difficult to arrange. Quantum cosmology, employing OQT, is not possible. (g) For somewhat similar reasons, OQT is such that it resists unification with general relativity. Such a unification would presumably involve attributing some kind of quantum state to spacetime itself (general relativity being a theory of spacetime). But, granted the basic structure of OQT, this would require that preparation and measurement devices exist outside spacetime, again not easy to arrange.

These nine defects, the two basic failures with which we began and the seven consequential defects, (a) to (g), are, taken together, very serious indeed. Despite its immense empirical success, OQT must be declared to be an unacceptably defective theory. It is the best of theories, and the worst of theories. ${ }^{2}$

In opposition to this conclusion, it may be argued that all physical theories, even a classical theory such as Newtonian theory (NT), must call upon additional theory to be tested empirically. In testing predictions of NT concerning the position of a planet at such and such a time, optical theory is required to predict the results of telescopic observations made here on earth. But this objection misses the point. NT is perfectly capable of issuing in physical predictions without calling upon additional theory, just because it has its own physical ontology. NT, plus initial and boundary conditions

2 Rival interpretations of quantum theory include: Bohm's interpretation, according to which quantum systems are both particles and waves; Everett's many-worlds interpretation; decoherence; consistent histories. None of these, in my view, provides us with a satisfactory version of quantum theory. For critical surveys and further literature see Squires (1986); Rae (2002, ch. 13); Bacciagaluppi (2003). 
formulated in terms of the theory, can issue in the physical prediction that such and such a planet is at such and such a place at such and such a time, whether anyone observes the planet or not, without calling upon optical theory or any other theory. This OQT cannot do. It cannot do this because it lacks its own quantum ontology, having failed to solve the quantum wave/particle problem. In order to deliver an unconditional physical prediction, OQT must call upon some part of classical physics, as a matter of necessity, so that the theory can refer to something physically actual. The case of NT and OQT are quite different, because NT postulates actually existing physical bodies whether observed or not, whereas QP does not; for that one requires OQT, that is QP + CP.

It may be objected that even if non-relativistic quantum theory fails to solve the wave/particle problem, relativistic quantum theory, or quantum field theory, does solve the problem in that it declares that what exists is the quantum field, "particles" being discrete excitations of the field. But this objection misses the point as well. Orthodox quantum field theory (OQFT) is just as dependent on measurement, and thus on some part of classical physics, as non-relativistic OQT is. The quantum states of the quantum field of OQFT have to be interpreted as making probabilistic predictions about the results of performing measurements, just as in the case of OQT. A version of quantum field theory which succeeded in specifying the nature of the quantum field in a fully satisfactory way, so that the theory has its own quantum ontology entirely independent of any part of classical physics, would be able to issue in physical predictions about actual physical states of affairs entirely independently of measurement. Such a theory would be able to predict and explain macroscopic, quasi-classical phenomena as arising from the quantum field alone, without calling upon some part of classical physics for a treatment of measurement. This OQFT cannot do.

\section{Probabilism to the rescue}

What needs to be done to cure OQT of its serious defects? The primary task must be to specify precisely and unambiguously the nature of quantum entities so that quantum theory (QT) can be formulated as a testable theory about how these entities evolve and interact without there being any mention of measurement or observables in the postulates of the theory at all. The key point that needs to be appreciated, I suggest, in order successfully to complete this task, is that the quantum domain is fundamentally probabilistic. $^{3}$ It is this that the manifestly probabilistic character of QT is trying to tell us.

The approach to solving the mysteries of the quantum domain that I am suggesting here has been long ignored largely because of the accidents of history. When Quantum Theory (QT) was being developed and interpreted, during the first three

\footnotetext{
${ }^{3}$ Popper has suggested that probabilism is the key to understanding wave/particle duality, and has put forward a propensity interpretation of quantum theory: see Popper $(1957,1967,1982)$. His interpretation of quantum theory is, however, unsatisfactory and quite different from the one I advocate here. For my criticisms of Popper see Maxwell (1976, 285-6; 1985, 41-2).
} 
decades of the last century, two opposing camps developed: the Bohr-Heisenberg camp, which argued for the abandonment of micro-realism, and the abandonment of determinism; and the Einstein-Schrödinger camp, which argued for the retention of realism, and the retention of determinism. One result of this polarization of views was that the idea of retaining realism but abandoning probabilism got overlooked. But it is just this overlooked option, I maintain, which gives us our best hope of curing the defects of QT. One might call this option probabilistic micro-realism.

Once we acknowledge that the quantum domain is fundamentally probabilistic, so that the basic laws governing the way quantum systems interact with one another are probabilistic, it is clear that measurement cannot be a satisfactory necessary and sufficient condition for probabilistic transitions to occur. Probabilistic transitions must be occurring in nature whether or not physicists are around to dub certain processes "measurements". The very notion of measurement is in any case, as we have seen, inherently imprecise. We require a new, precise, necessary and sufficient condition for probabilistic transitions to occur, to be specified in fundamental, quantum mechanical terms.

Furthermore, once the fundamentally probabilistic character of the quantum domain is acknowledged, it immediately becomes clear how the key quantum wave/particle problem is to be solved. If the quantum domain is fundamentally probabilistic, then the physical entities of this domain, electrons, atoms and the rest, cannot possibly be classical, deterministic entities — classical particles, waves or fields. Quite generally, we should hold that there is a one-to-one correspondence between the dynamical laws of a physical theory on the one hand, and the entities and their physical properties postulated by the theory, on the other hand. In speaking of the entities, and the properties of entities, postulated by a physical theory, we are thereby speaking, in other terms, of the dynamical laws of the theory. Hence, change dynamical laws in some basic way, and we thereby change postulated physical entities and their properties. In particular, change dynamical laws dramatically, so that they become probabilistic instead of being deterministic, and the nature of postulated physical entities must change dramatically as well. Quantum entities, interacting with one another probabilistically, must be quite different from all physical entities so far encountered within deterministic classical physics.

[Elsewhere (Maxwell, 1976, 283-6; 1988, 44-8) I have indicated how the notion of probabilistic physical property, or propensity, that is being presupposed here, amounts to a probabilistic generalization of the notion of deterministic, necessitating property explicated in Maxwell (1968); see also Maxwell (1998, 141-55). I might add, no doubt controversially, that in my view my 1968 paper gives the definitive account of how dispositional, necessitating properties in physics should be conceived. This viewpoint, in particular, makes no appeal to Kripke's (1981) fallacious considerations concerning identity and necessity: for a refutation of Kripke, see Maxwell (2001, appendix 2). Much subsequent work on dispositional properties in science is vitiated by a failure to take my earlier work into account, and a reliance instead on Kripke.] 
The defects of OQT have arisen, in other words, because physicists have sought to interpret probabilistic quantum theory in terms of classical waves and particles, deterministic metaphysical ideas appropriate to earlier classical physics but wholly inappropriate to the new quantum theory. The failure of this entirely misguided attempt then led to despair at the possibility of solving the (misconstrued) wave/particle problem, despair at the possibility of specifying the precise physical nature of quantum entities. This despair in turn led to the development of OQT as a theory about the results of performing measurements —a theory which, it seemed, did not need to specify the precise nature of quantum entities. But the outcome is a theory burdened with the nine serious defects indicated above.

Thus the traditional quantum wave/particle problem is the wrong problem to pose. We should ask, not "Are quantum entities waves or particles?", but rather (1) What kinds of possible, unproblematic, fundamentally probabilistic physical entities are there?, and (2) Are quantum entities one kind of such unproblematic probabilistic entity?

The failure to put right the serious defects of OQT has persisted for so long because physicists have abandoned hope of solving the traditional quantum wave/particle problem, not realizing that this is entirely the wrong problem to try to solve in the first place. Once it is appreciated that (1) and (2) are the right problems to try to solve, new possibilities, long overlooked, immediately spring to mind.

First, physical entities that interact with one another probabilistically may be dubbed propensitons. Two kinds of unproblematic propensiton can immediately be distinguished: continuous propensitons, which evolve probabilistically continuously in time, and intermittent propensitons, which evolve deterministically except for intermittent moments in time when appropriate physical conditions arise, and the propensitons undergo probabilistic transitions.

There is a second obvious distinction that can be made between propensitons which spread out spatially in time, increasing the volume of space they occupy with the passage of time, and propensitons which do not spread spatially in this way. Let us call the first spatially spreading propensitons, and the second spatially confined propensitons.

We are in new territory. In our ordinary experience of the world, and within deterministic physics, we never encounter propensitons. Probabilistic outcomes, obtained when we toss a penny or a die, can always be put down to probabilistic changes in initial conditions. Classical statistical mechanics presupposes that the underlying dynamic laws are deterministic. Having no experience of them, propensitons will, inevitably, when we first encounter them, strike us as mysterious, even unacceptably weird. But these feelings of unfamiliarity ought not to lead us into deciding that theories which postulate such entities are inherently unacceptable. In particular, the four kinds of propensity indicated above should be regarded as equally viable, a priori. Whether a theory that postulates one or other type of propensiton is acceptable or not should be decided upon in the usual way, in terms of its empirical success, and the extent to which it is unified, simple, explanatory. 
Granted that quantum systems are some kind of propensiton, which of the four kinds of unproblematic propensiton just indicated should we take quantum systems to be? There is here a very important consideration to be borne in mind. Despite suffering from the nine defects indicated above, nevertheless OQT is perhaps the most empirically successful physical theory ever formulated. The range, variety and accuracy of its empirical predictions are unprecedented. No other physical theory has been subjected to such sustained severe experimental testing, and has survived without a single refutation. There are good grounds for holding that OQT has got quite a lot right about the nature of the quantum world. Our strategy, then, ought to be, in the first instance at least, to stick as close to OQT as possible, and modify OQT just sufficiently to remove the defects of the theory. The structure of OQT mirrors that of the intermittent, spatially spreading propensiton. On the one hand quantum states evolve deterministically, in accordance with Schrödinger's time-dependent equation; on the other hand, there are, on the face of it, probabilistic transitions associated with measurement. Quantum states spread out spatially when evolving deterministically, and tend to become localized when measurements are made. All this mirrors the character of the intermittent, spatially spreading propensiton, the only unsatisfactory feature of OQT being that the theory stipulates that probabilistic transitions occur when measurements are made.

A very elementary kind of spatially spreading intermittent propensiton is the following. It consists of a sphere, which expands at a steady rate (deterministic evolution) until it touches a second sphere, at which moment the sphere becomes instantaneously a minute sphere, of definite radius, somewhere within the space occupied by the large sphere, probabilistically determined. The second sphere undergoes the same instantaneous probabilistic transition. Then both minute spheres again undergo steady, deterministic expansion, until they touch once more, and another probabilistic localization occurs.

A slightly more sophisticated version of this elementary spatially spreading intermittent propensiton is the following. The sphere is made up of variable "position probability density", such that, when the sphere localizes probabilistically, in the way just indicated, it is most probable that it will be localized where the position probability density is most dense. A law specifies how position probability density is distributed throughout the sphere. We might even imagine that the position probability density exhibits a wave-like distribution. Such a propensiton, given appropriate conditions for probabilistic localization, might even exhibit interference phenomena in a two-slit experiment!

Quantum entities, such as electrons, photons and atoms, are, I suggest, spatially spreading intermittent propensitons. Their physical state is specified by the $\Psi$ function of QT. The deterministic evolution of these quantum propensitons is specified by Schrödinger's time-dependent equation:

$$
\text { ih } \frac{\partial \Phi(\mathrm{t})}{\partial \mathrm{t}}=-\frac{\mathbf{h}^{2}}{2 \mathrm{~m}} \nabla^{2} \Phi(\mathrm{t})+\mathrm{V} \Phi(\mathrm{t})
$$


The crucial questions that need to be answered to specify precisely the probabilistic properties —or propensities — of quantum systems are these:

(a) What is the precise quantum mechanical condition for a probabilistic transition to occur?

(b) Given the quantum state, $\Psi$, at the instant before the probabilistic transition, how does this determine what the possible outcome states are, $\phi_{1}, \phi_{2}$, $\ldots \phi_{\mathrm{N}}$ ?

(c) How does $\Psi$ determine the probability $p_{r}$ that the outcome of the probabilistic transition will be $\phi_{\mathrm{r}}$, for $\mathrm{r}=1,2, \ldots \mathrm{N}$ ?

(d) How can (a) to (c) be answered so that the resulting fundamentally probabilistic version of quantum theory reproduces all the empirical success of OQT?

A number of different answers can be given to (a) to (d).

One possibility is the proposal of Ghirardi, Rimini and Weber (see Ghirardi and Rimini, 1990) according to which the quantum state of a system such as an electron collapses spontaneously, on average after the passage of millions of years, into a highly localized state. When a measurement is performed on the quantum system, it becomes quantum entangled with millions upon millions of quantum systems that go to make up the measuring apparatus. In a very short time there is a high probability that one of these quantum systems will spontaneously collapse, causing all the other quantum entangled systems, including the electron, to collapse as well. At the micro level, it is almost impossible to detect collapse, but at the macro level, associated with measurement, collapse occurs very rapidly all the time.

Another possibility is the proposal of Penrose (1986), according to which collapse occurs when the state of a system evolves into a superposition of two or more states, each state having, associated with it, a sufficiently large mass located at a distinct region of space. The idea is that general relativity imposes a restriction on the extent to which such superpositions can develop, in that it does not permit such superpositions to evolve to such an extent that each state of the superposition has a substantially distinct space-time curvature associated with it.

The possibility that I favour, put forward before either Ghirardi, Rimini and Weber's proposal, or Penrose's proposal, is that probabilistic transitions occur whenever, as a result of inelastic interactions between quantum systems, new "particles", new bound or stationary systems, are created (Maxwell, 1972, 1976, 1982, 1988, 1994). A little more precisely:

Postulate $1 \mathrm{~A}$ : Whenever, as a result of an inelastic interaction, a system of interacting "particles" creates new "particles", bound or stationary systems, so that the state of the system goes into a superposition of states, each state having associated with it different particles or bound or stationary systems, then, when the interaction is nearly at an end, spontaneously and probabilistically, entirely 
in the absence of measurement, the superposition collapses into one or other state.

Two examples of the kind of interactions that are involved here are the following:

$$
\begin{aligned}
& \mathrm{e}^{-}+\mathrm{H} \\
& \mathrm{e}^{-}+\mathrm{H} \rightarrow \mathrm{e}^{-}+\mathrm{H}^{*} \\
& \mathrm{e}^{-}+\mathrm{H}+\gamma \\
& \mathrm{e}^{-}+\mathrm{e}^{-}+\mathrm{p} \\
& \mathrm{e}^{+}+\mathrm{H} \\
& \mathrm{e}^{+}+\mathrm{H} \rightarrow \mathrm{e}^{+}+\mathrm{e}^{-}+\mathrm{p} \\
& \left(\mathrm{e}^{+} / \mathrm{e}^{-}\right)+\mathrm{p} \\
& \mathrm{p}+2 \gamma
\end{aligned}
$$

(Here $\mathrm{e}^{-}, \mathrm{e}^{+}, \mathrm{H}, \mathrm{H}^{*}, \gamma, \mathrm{p}$ and $\left(\mathrm{e}^{+} / \mathrm{e}^{-}\right)$stand for electron, positron, hydrogen atom, excited hydrogen atom, photon, proton and bound system of electron and positron, respectively.)

What exactly does it mean to say that the "interaction is very nearly at an end" in the above postulate? My suggestion, here, is that it means that forces between the "particles" are very nearly zero, except for forces holding bound systems together. In order to indicate how this can be formulated precisely, consider the toy interaction:

$$
\begin{aligned}
a+b+c \rightarrow & a+b+c \\
a & +(b c)
\end{aligned}
$$

Here, $a, b$ and $\mathrm{c}$ are spinless particles, and (bc) is the bound system. Let the state of the entire system be $\Phi(t)$, and let the asymptotic states of the two channels (A) and (B) be $\psi_{\mathrm{A}}(\mathrm{t})$ and $\psi_{\mathrm{B}}(\mathrm{t})$ respectively. Asymptotic states associated with inelastic interactions are fictional states towards which, according to OQT, the real state of the system evolves as $t \rightarrow+\infty$. Each outcome channel has its associated asymptotic state, which evolves as if forces between particles are zero, except where forces hold bound systems together.

According to OQT, in connection with the toy interaction above, there are states $\phi_{\mathrm{A}}(\mathrm{t})$ and $\phi_{\mathrm{B}}(\mathrm{t})$ such that:

(1) For all $t, \Phi(t)=c_{A} \phi_{A}(t)+c_{B} \phi_{B}(t)$, with $\left|c_{A}\right|^{2}+\left|c_{B}\right|^{2}=1$;

(2) as $\mathrm{t} \rightarrow+\infty, \phi_{\mathrm{A}}(\mathrm{t}) \rightarrow \psi_{\mathrm{A}}(\mathrm{t})$ and $\phi_{\mathrm{B}}(\mathrm{t}) \rightarrow \psi_{\mathrm{B}}(\mathrm{t})$.

The idea is that at the first instant $t$ for which $\phi_{A}(t)$ is very nearly the same as the asymptotic state $\psi_{\mathrm{A}}(\mathrm{t})$, and $\phi_{\mathrm{B}}(\mathrm{t})$ is very nearly the same as $\psi_{\mathrm{B}}(\mathrm{t})$, then the state of the system, $\Phi(t)$, collapses spontaneously either into $\phi_{A}(t)$ with probability $\left|c_{A}\right|^{2}$, or into $\phi_{\mathrm{B}}(\mathrm{t})$ with probability $\left|\mathrm{c}_{\mathrm{B}}\right|^{2}$. Or, more precisely:

Postulate 1B: At the first instant for which $|\langle\psi \mathrm{A}(\mathrm{t}) \mid \phi \mathrm{A}(\mathrm{t})\rangle| 2>1-\varepsilon$ or 
$|\langle\psi \mathrm{B}(\mathrm{t}) \mid \phi \mathrm{B}(\mathrm{t})\rangle| 2>1-\varepsilon$, the state of the system collapses spontaneously into $\phi \mathrm{A}(\mathrm{t})$ with probability $|\mathrm{cA}| 2$, or into $\phi \mathrm{B}(\mathrm{t})$ with probability $|\mathrm{cB}| 2, \varepsilon$ being a universal constant, a positive real number very nearly equal to zero.

The evolutions of the actual state of the system, $\Phi(\mathrm{t})$, and the asymptotic states, $\psi_{A}(t)$ and $\psi_{B}(t)$, are governed by the respective channel Hamiltonians, $\mathrm{H}, \mathrm{H}_{\mathrm{A}}$ and $\mathrm{H}_{\mathrm{B}}$, where:

$$
\begin{aligned}
& \mathrm{H}=-\left(\frac{\mathbf{h}^{2}}{2 \mathrm{~m}_{\mathrm{a}}} \nabla_{\mathrm{a}}^{2}+\frac{\mathbf{h}^{2}}{2 \mathrm{~m}_{\mathrm{b}}} \nabla_{\mathrm{b}}^{2}+\frac{\mathbf{h}^{2}}{2 \mathrm{~m}_{\mathrm{c}}} \nabla_{\mathrm{c}^{2}}\right)+\mathrm{V}_{\mathrm{ab}}+\mathrm{V}_{\mathrm{ac}}+\mathrm{V}_{\mathrm{ac}} \\
& \mathrm{H}_{\mathrm{A}}=-\left(\frac{\mathbf{h}^{2}}{2 \mathrm{~m}_{\mathrm{a}}} \nabla_{\mathrm{a}}^{2}+\frac{\mathbf{h}^{2}}{2 \mathrm{~m}_{\mathrm{b}}} \nabla_{\mathrm{b}^{2}}+\frac{\mathbf{h}^{2}}{2 \mathrm{~m}_{\mathrm{c}}} \nabla_{\mathrm{c}^{2}}\right) \\
& \mathrm{H}_{\mathrm{B}}=-\left(\frac{\mathbf{h}^{2}}{2 \mathrm{~m}_{\mathrm{a}}} \nabla_{\mathrm{a}}^{2}+\frac{\boldsymbol{h}^{2}}{2 \mathrm{~m}_{\mathrm{b}}} \nabla_{b^{2}}+\frac{\mathbf{h}^{2}}{2 \mathrm{~m}_{\mathrm{c}}} \nabla_{\mathrm{c}^{2}}\right)+\mathrm{V}_{\mathrm{bc}}
\end{aligned}
$$

Here, $\mathrm{m}_{\mathrm{a}}, \mathrm{m}_{\mathrm{b}}$, and $\mathrm{m}_{\mathrm{c}}$ are the masses of "particles" $\mathrm{a}, \mathrm{b}$ and $\mathrm{c}$ respectively, and $\mathbf{h}=$ $\mathrm{h} / 2 \pi$ where $\mathrm{h}$ is Planck's constant.

The condition for probabilistic collapse, formulated above, can readily be generalized to apply to more complicated and realistic inelastic interactions between "particles".

According to the micro-realistic, fundamentally probabilistic version of quantum theory, indicated above, the state function, $\Phi(\mathrm{t})$, describes the actual physical state of the quantum system, from moment to moment. Quantum systems may be called "propensitons". The physical (quantum) state of the propensiton evolves in accordance with Schrödinger's time-dependent equation as long as the condition for a probabilistic transition to occur does not obtain. The moment it does obtain, the state jumps instantaneously and probabilistically, in the manner indicated above, into a new state. (All but one of a superposition of states, each with distinct "particles" associated with them, vanish.) The new state then continues to evolve in accordance Schrödinger's equation until conditions for a new probabilistic transition arise.

Propensiton quantum theory (PQT), as we may call this micro-realistic, fundamentally probabilistic version of quantum theory, can recover all the experimental success of OQT. This follows from four points. First, OQT and PQT use the same dynamical equation, namely Schrödinger's time-dependent equation. Secondly, whenever a position measurement is made, and a quantum system is detected, this invariably involves the creation of a new "particle" (bound or stationary system, such as the ionisation of an atom or the dissociation of a molecule, usually millions of these). This means that whenever a position measurement is made, the conditions for probabilistic transitions to occur, according to PQT, are satisfied. PQT will reproduce the predictions of OQT (given that PQT is provided with a specification of the quantum state of the measur- 
ing apparatus). Thirdly, all other observables of OQT, such as momentum, energy, angular momentum or spin, always involve (i) a preparation procedure which leads to distinct spatial locations being associated with distinct values of the observable to be measured, and (ii) a position measurement in one or other spatial location. This means that PQT can predict the outcome of measurements of all the observables of OQT. Fourthly, insofar as the predictions of OQT and PQT differ, the difference is extraordinarily difficult to detect, and will not be detectable in any quantum measurement so far performed.

In principle, however, OQT and PQT yield predictions that differ for experiments that are extraordinarily difficult to perform, and which have not yet, to my knowledge, been performed. Consider the following evolution:

$$
\mathrm{a}+\mathrm{b}+\mathrm{c} \longrightarrow \begin{gathered}
\text { collision } \\
\mathrm{a}+\mathrm{b}+\mathrm{c} \\
\mathrm{a}+\mathrm{bc})
\end{gathered} \longrightarrow \mathrm{a}+\mathrm{b}+\mathrm{c}
$$

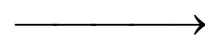

Suppose the experimental arrangement is such that, if the superposition at stage (3) persists, then interference effects will be detected at stage (5). Suppose, now, that at stage (3) the condition for the superposition to collapse into one or other state, according to PQT, obtains. In these circumstances, OQT predicts interference at stage (5), whereas PQT predicts no interference at stage (5), (assuming the above evolution is repeated many times). PQT predicts that in each individual case, at stage (3), the superposition collapses probabilistically into one or other state. Hence there can be no interference.

\section{Further questions}

It may be asked how $\Phi(\mathrm{t})$ can possibly represent the real physical state of a quantum system given that $\Phi(\mathrm{t})$ is a complex function of space and time. The answer is that $\Phi(t)$ can always be construed to depict two real functions of space and time.

It may be asked how $\Phi(\mathrm{t})$ can possibly represent the real physical state of a quantum system consisting of two (or more) quantum entangled "particles", since in this case $\Phi(\mathrm{t})$ is a function of six dimensional configuration space plus time (or, in general, a function of $3 \mathrm{~N}$ configuration space plus time, where $\mathrm{N}$ is the number of quantum entangled "particles" that go to make up the system in question). In the case of two "particles", we can construe $\Phi\left(r_{1}, r_{2}, t\right)$, where $r_{1}$ and $r_{2}$ are the spatial coordinates of "particles" 1 and 2 respectively, as depicting the propensity state of the system in real 3-dimensional physical space, as follows. $\left|\Phi\left(r_{1}, r_{2}, t\right)\right|^{2} d V_{1} d V_{2}$ represents the probability of the system interacting in a localizing (wave-packet-collapsing) way such that "particle" 1 interacts in volume element $\mathrm{dV}_{1}$ about spatial coordinates $r_{1}$, and "parti- 
cle" 2 interacts in volume element $\mathrm{dV}_{2}$ about spatial coordinates $\mathrm{r}_{2}$. The quantum entangled nature of the system means that as $r_{2}$ is changed, so the probability of "particle" 1 interacting in $\mathrm{dV}_{1}$ about $\mathrm{r}_{2}$ will, in general, change too.

It may be objected that postulate $1(\mathrm{~A}+\mathrm{B})$ provides no mechanism for quantum systems to be localized. This is not correct. If a highly localized system, $\mathrm{S}_{1}$, interacts inelastically with a highly unlocalized system, $\mathrm{S}_{2}$, in such a way that a probabilistic transition occurs, then $S_{1}$ will localize $S_{2}$. If an atom or nucleus emits a photon which travels outwards in a spherical shell and which is subsequently absorbed by a localized third system, the localization of the photon will localize the emitting atom or nucleus with which it was quantum entangled.

Postulate $1(\mathrm{~A}+\mathrm{B})$ above has been formulated for rearrangement collisions. But the postulate is intended to apply to inelastic interactions that lead to the creation (or annihilation) of new particles, as in interactions such as $\mathrm{e}^{-}+\mathrm{e}^{+} \rightarrow 2 \gamma$. Such interactions require that one employs relativistic QT, which is beyond the scope of the present paper. It deserves to be noted, however, that the root idea that probabilistic transitions occur when new "particles" are created can be interpreted in a number of different ways.

(1) There is the option considered above. The inelastic interaction must be such that distinct "particle" channels have, associated with them, distinct asymptotic states which evolve in accordance with distinct Hamiltonians. This means at least that distinct "particles" have different masses associated with them (so that an excited state of a bound system is, potentially, a different "particle" from the ground state, since the excited state will be slightly more massive than the ground state).

(2) As above, except that, for two interaction channels to differ it is not sufficient that "particles" associated with the two channels have distinct masses; either there are different numbers of "particles" (counting a bound system as one "particle") associated with different channels, or there is at least one "particle" which has a different charge, or force, associated with it.

(3) For a probabilistic transition to occur, rest mass must be converted into energy of "particles" without rest mass (eg photons), or vice versa.

(4) For a probabilistic transition to occur, fermions must be converted into bosons, or vice versa.

Only experiment can decide between these options. The import of this paper, and of previous papers published by the author (Maxwell, 1972; 1973a; 1973b; 1976; 1982; $1988 ; 1993 b ; 1994 ; 1995 ; 1998$, ch. 7) is that a major research effort ought to get underway, both theoretical and experimental, devoted to exploring and testing rival collapse hypotheses. Only in this way will a version of quantum theory be developed free of the defects of OQT which also meets with greater empirical success than OQT. Only in this way will physics succeed in providing some kind of answer to the two childishly elementary, inter-related questions with which we began. 


\section{Quantum confusions a part of a historical pattern}

I conclude with a historical remark. I have argued that the long-standing failure to solve the mysteries of the quantum domain - and so to develop a fully acceptable version of quantum theory- is due to the misguided attempt to understand the probabilistic quantum domain in terms of deterministic metaphysical ideas appropriate to the earlier theories of classical physics. As a result of the failure to solve the wholly misguided traditional wave/particle problem, Heisenberg, Bohr, Born and others developed quantum theory as a theory about the results of performing measurements, which seemed successfully to avoid the need to specify precisely the nature of quantum systems, but which unintentionally led to the creation of a theory with severe, if somewhat surreptitious, defects.

This pattern of confusion has occurred on at least two earlier occasions in the history of physics. On these occasions, too, physicists have attempted to interpret a new theory in terms of old, inappropriate metaphysics; the failure of this misguided effort then leads to despair at the possibility of interpreting the new theory realistically. It leads to instrumentalism, in other words, to the view that physical theories have to be interpreted as being about observable phenomena, and not about unobservable physical entities such as particles and fields. Eventually, however, the new theory may be interpreted in terms of new appropriate metaphysics. Physicists, one might say, are brilliant when it comes to equations, but not so brilliant — or at least very conservativewhen it comes to metaphysics.

An example is Newton's theory of gravitation which postulates a force at a distance between bodies with mass. The reigning metaphysical idea at the time was the corpuscular hypothesis, the thesis that nature is made up of tiny corpuscles which interact only by contact. This thesis functioned as a standard of intelligibility: no fundamental physical theory could claim to be intelligible if it could not be interpreted in terms of the corpuscular hypothesis. The impossibility of interpreting Newton's theory of gravitation in terms of the corpuscular hypothesis initially led some of Newton's most eminent contemporaries to reject Newton's theory. Thus Huygens, in a letter to Leibniz, writes: "Concerning the Cause of the flux given by M. Newton, I am by no means satisfied [by it], nor by all the other Theories that he builds upon his Principle of Attraction, which seems to me absurd... I have often wondered how he could have given himself all the trouble of making such a number of investigations and difficult calculations that have no other foundation that this very principle" (Koyre, 1965, pp. 117-8). Newton in a sense agreed, as is indicated by his remark: "That gravity should be innate, inherent and essential to matter, so that one body may act upon another, at a distance through a vacuum, without the mediation of anything else... is to me so great an absurdity, that I believe no man who has in philosophical matters a competent faculty of thinking can ever fall into it" (Burtt, 1932, pp. 265-6). The impossibility of interpreting the law of gravitation in terms of the corpuscular hypothesis, in terms of action-by-contact, led Newton to interpret the law instrumentalistically, as specifying the way bodies move without providing any kind of explanation for the motion, in terms of unobservable forces. Subsequently, however, Boscovich and oth- 
ers were able to conceive of a metaphysical view more appropriate to Newton's new theory, according to which nature is made up of point-particles, with mass, each point-particle being surrounded by a rigid, spherically-symmetric, centrally directed field of force which varies with distance. Reject the corpuscular hypothesis and adopt, instead, this new Boscovichean metaphysics, and Newton's theory ceases to be incomprehensible, and becomes the very model of comprehensibility.

Another example is provided by James Clerk Maxwell's theory of electrodynamics. Maxwell himself, and most of his contemporaries and immediate successors, sought to interpret the electromagnetic field in terms of a material substratum, the hypothetical aether, itself to be understood in Newtonian terms. A tremendous amount of effort was put into trying to understand Maxwell's field equations in terms of the aether. Faraday, who appreciated that one should take the electromagnetic field as a new kind of physical entity, and explain matter in terms of the field rather than try to explain the field in terms of a kind of hypothetical matter (the aether), was ignored. The unrealistic character, and ultimate failure, of mechanical models of the electromagnetic field led many to hold that the real nature of the field must remain a mystery. The most that one could hope for from Maxwell's equations, it seemed, was the successful prediction of observable phenomena associated with electromagnetism. This instrumentalistic attitude remained even after the advent of Einstein's special theory of relativity in 1905, which might be interpreted as giving credence to the idea that it is the field that is fundamental. Gradually, however, Einstein and others came to adopt the view that one should see the field as a new kind of physical entity, quite distinct from corpuscle and point-particle.

There are two lessons to be learned from these episodes, one for quantum theory specifically, the other for theoretical physics in general. In the first place, quantum theory, if fundamentally probabilistic, needs to be formulated as a theory about fundamentally probabilistic physical entities — propensitons - however weird these may seem given our common sense and classical intuitions. We require a fully microrealistic version of quantum theory which, though testable, says nothing about "observables" or "measurement" in the basic postulates of the theory at all. Secondly, if theoretical physics is to free itself from the obstructive tendency to interpret new theories in terms of old, inappropriate metaphysics, physicists need to recognize that metaphysical ideas are inevitably an integral part of theoretical physics, and need to be developed and improved in the light of new theoretical developments. Elsewhere (Maxwell, 1998), I have argued that, in order to construe physics as a rational enterprise, we need to see physics as making a hierarchy of metaphysical assumptions concerning the comprehensibility and knowability of the universe, these assumptions becoming increasingly insubstantial, and thus increasingly likely to be true, as we ascend the hierarchy. According to this "aim-oriented empiricist" view, this hierarchy creates a framework of reasonably secure, permanent assumptions (and associated methods) within which much more specific and fallible assumptions (and associated methods), low down in the hierarchy, can be revised and improved. If ever the physics community came to accept and put into scientific practice this aim-oriented empiricist meth- 
odology, then the best available metaphysical ideas might lead the way to the discovery of new physical theories, instead of obstructing interpretation and understanding of theories that have been discovered (and thus also obstructing the discovery of new theories). In one exceptional case in the history of physics, the new metaphysics came first, led the way, and actually made possible the subsequent discovery of the new theory. This happened when Einstein discovered general relativity. Einstein first hit upon the metaphysical idea that gravitation is due to the curvature of space-time, and then subsequently discovered how to capture this idea precisely in the field equations of general relativity. In stark contrast to the cases of Newtonian theory, Maxwellian classical electrodynamics and quantum theory, general relativity was discovered as a result of the prior development of new appropriate metaphysics, instead of the discovery of the new theory, if anything, being obstructed by current metaphysical ideas, the theory being misunderstood and misinterpreted by such ideas, once discovered. That Einstein's discovery of general relativity should stand out in this way is not, in my view, surprising: as I have argued elsewhere (Maxwell 1993a), Einstein both put into practice, and upheld, a conception of science close to that of aim-oriented empiricism.

\section{REFERENCES}

Bacciagaluppi, G. (2003). "The Role of Decoherence in Quantum Mechanics”, The Stanford Encyclopedia of Philosophy (Winter 2003 Edition), Edward N. Zalta (ed.), URL = <http://plato.stanford.edu/archives/ win2003/entries/qm-decoherence/>.

Burtt, E.A. (1932). The Metaphysical Foundations of Modern Science. London: Routledge and Kegan Paul.

Feynman, R.P., et al. (1965). The Feynman Lectures on Physics. Reading (Mass.): Addison-Wesley.

Ghirardi, G.C., and A. Rimini (1990). "Old and New Ideas in the Theory of Quantum Measurement", in A. Miller (ed.), Sixty-Two Years of Uncertainty. New York: Plenum Press, pp. 167-91.

Gillespie, T.D. (1973). A Quantum Mechanical Primer. Aylesbury: International Textbook.

Koyre, A. (1965). Newtonian Studies. Chapman and Hall.

Kripke, S. (1981). Naming and Necessity. Oxford: Blackwell.

Maxwell, N. (1968). "Can there be Necessary Connections between Successive Events?”, British Journal for the Philosophy of Science 19, 295-311.

- (1972). "A New Look at the Quantum Mechanical Problem of Measurement", American Journal of Physics 40, pp. 1431-5.

_ (1973a). "Alpha Particle Emission and the Orthodox Interpretation of Quantum Mechanics", Physics Letters 43A, pp. 29-30.

- (1973b). "The Problem of Measurement - Real or Imaginary?", American Journal of Physics 41, pp. 1022-5.

(1976). “Towards a Micro Realistic Version of Quantum Mechanics, Parts I and II", Foundations of Physics 6, pp. 275-92 and 661-76.

(1982). "Instead of Particles and Fields", Foundations of Physics 12, 607-31.

(1988). "Quantum Propensiton Theory: A Testable Resolution of the Wave/Particle Dilemma", British Journal for the Philosophy of Science 39, 1-50.

_ (1993a). "Induction and Scientific Realism: Einstein, Aim-Oriented Empiricism and the Discovery of Special and General Relativity", The British Journal for the Philosophy of Science 44, 1993, 275-305.

- (1993b). "Beyond Fapp: Three Approaches to Improving Orthodox Quantum Theory and An Experimental Test", in A. van der Merwe, F. Selleri and G. Tarozzi (eds.), Bell's Theorem and the Foundations of Modern Physics. World Scientific, pp. 362-370. 
(1994), "Particle Creation as the Quantum Condition for Probabilistic Events to Occur", Physics Letters $A$ 187, 351-5.

(1995). "A Philosopher Struggles to Understand Quantum Theory: Particle Creation and Wavepacket Reduction", in M. Ferrero and A. van der Merwe (eds.), Fundamental Problems in Quantum Physics. London: Kluwer Academic, pp. 205-214.

- (1998). The Comprehensibility of the Universe: A New Conception of Science. Oxford: Oxford University Press, (paperback 2003).

- (2001). The Human World in the Physical Universe: Consciousness, Free Will and Evolution. Lanham: Rowman and Littlefield.

Penrose, R. (1986). "Gravity and State Reduction", in R. Penrose and C. J. Isham (eds.), Quantum Concepts of Space and Time. Oxford: Oxford University Press, pp. 129-146.

Popper, K.R. (1957). "The Propensity Interpretation of the Calculus of Probability and the Quantum Theory", in S. Körner (ed.), Observation and Interpretation. London: Butterworth, pp. 65-70.

— (1967). "Quantum Mechanics without 'The Observer", in M. Bunge(ed.), Quantum Theory and Reality. Berlin: Springer, pp. 7-44.

- (1982). Quantum Theory and the Schism in Physics. London: Hutchinson.

Rae, A.I.M. (2002). Quantum Mechanics. Bristol: Institute of Physics Publishing.

Squires, E. (1986). The Mystery of the Quantum World. Bristol: Adam Hilger.

Nicholas Maxwell is Emeritus Reader in Philosophy of Science at University College London. He has devoted much of his working life to trying to get across the idea that we need a revolution in academic inquiry so that the basic aim becomes to promote wisdom and not just acquire knowledge. His books include From Knowledge to Wisdom (Blackwell, 1984), The Comprehensibility of the Universe (OUP, 1998), The Human World in the Physical Universe (Rowman and Littlefield, 2001), and Is Science Neurotic? (Imperial College Press, November 2004).

AdDREss: University College London. Email: nicholas.maxwell@ucl.ac.uk. 